\title{
Quality-adjusted survival with combination nal-IRI + 5-FU/LV vs 5-FU/LV alone in metastatic pancreatic cancer patients previously treated with gemcitabine-based therapy: a Q-TWiST analysis
}

Uwe Pelzer ${ }^{1}$, Jean-Frédéric Blanc ${ }^{2}$, Davide Melisi ${ }^{3}$, Antonio Cubillo ${ }^{4}$, Daniel D Von Hoff ${ }^{5}$, Andrea Wang-Gillam ${ }^{6}$, Li-Tzong Chen ${ }^{7}$, Jens T Siveke ${ }^{8,9}$, Yin Wan ${ }^{10}$, Caitlyn T Solem ${ }^{10}$, Marc F Botteman ${ }^{10}$, Yoojung Yang ${ }^{11}$, Floris A de Jong ${ }^{12}$ and Richard A Hubner ${ }^{13}$

${ }^{1}$ Department of Hematology/Oncology/Tumorimmunology, Charité - Universitätsmedizin Berlin, Augustenburger Platz 1, 13353 Berlin, Germany; ${ }^{2}$ Service d'Hépato-Gastroentérologie et Oncologie Digestive, Hôpital Haut-Lévêque, CHU de Bordeaux, Inserm UMR 1053, Université de Bordeaux, Bordeaux, France; ${ }^{3}$ Digestive Molecular Clinical Oncology Unit, University of Verona, Piazzale L.A. Scuro, 10, 37134 Verona, Italy; ${ }^{4}$ Servicio de Oncologia Médica, Centro Integral Oncológico Clara Campal (CIOCC), Hospital Universitario Madrid Sanchinarro, Calle Oña, 10, 28050 Madrid, Spain; ${ }^{5}$ Virginia G. Piper Cancer Center at HonorHealth/TGen, 10460 N 92nd St \#206, Scottsdale, AZ 85258, USA; ' ${ }^{6}$ Division of Oncology, Washington University in St Louis, 660 South Euclid Avenue, St Louis, MO 63110, USA; ${ }^{7}$ National Institute of Cancer Research, National Health Research Institutes, 2F, No. 367, Sheng-Li Road, Tainan 70456, Taiwan; ${ }^{8}$ Division of Solid Tumor Translational Oncology, DKTK Partner Site Essen, West German Cancer Center, University Hospital Essen, Hufelandstrasse 55, 45147 Essen, Germany; ${ }^{9}$ German Cancer Consortium (DKTK) and German Cancer Research Center (DKFZ), Heidelberg, Germany; ${ }^{10}$ Pharmerit International, 4350 East-West Hwy \#430, Bethesda, MD 20814, USA; ${ }^{11}$ Shire, Plc, 650 East Kendal St, Cambridge, MA 02142, USA; ${ }^{12}$ Department of Global Medical Affairs Oncology, Shire GmbH, Zählerweg 10, 6300 Zug, Switzerland and ${ }^{13}$ Department of Medical Oncology, Christie Hospital NHS Foundation Trust, 550 Wilmslow Rd, Manchester M20 4BX, UK

Background: In the NAPOLI-1 Phase 3 trial, nal-IRI + 5-fluorouracil and leucovorin (5-FU/LV) significantly improved median overall survival (6.1 vs 4.2 months, $P=0.012$ ) and progression-free survival (3.1 vs 1.5 months, $P=0.0001$ ) vs 5 -FU/LV alone in metastatic pancreatic adenocarcinoma patients previously treated with gemcitabine-based therapy. This analysis evaluated between treatment differences in quality-adjusted time without symptoms of disease progression or toxicity (Q-TWiST).

Methods: Overall survival was partitioned into time with grade $\geqslant 3$ toxicity (TOX), disease progression (REL), and time without disease progression symptoms or grade $\geqslant 3$ toxicity (TWiST). Mean Q-TWiST was calculated by weighting time spent by a utility of 1.0 for TWiST and 0.5 for TOX and REL. In threshold analyses, utility for TOX and REL were varied from 0.0 to 1.0.

Results: Patients in nal-IRI + 5-FU/LV ( $n=117)$ vs 5-FU/LV ( $n=119)$ had significantly more mean time in TWiST (3.4 vs 2.4 months) and TOX (1.0 vs 0.3 months) but similar REL (2.5 vs 2.7 months). In the base case, nal-IRI + 5-FU/LV patients had 1.3 months (95\% Cl, $0.4-2.1 ; 5.1$ vs 3.9) greater Q-TWiST (threshold analyses range: 0.9-1.6 months).

Conclusions: Within NAPOLI-1, nal-IRI + 5-FU/LV resulted in statistically significant and clinically meaningful gains in qualityadjusted survival vs 5-FU/LV alone.

*Correspondence: Dr U Pelzer; E-mail uwe.pelzer@charite.de

Received 8 July 2016; revised 31 January 2017; accepted 5 February 2017; published online 28 March 2017

(C) 2017 Cancer Research UK. All rights reserved 0007-0920/17 
Pancreatic cancer remains one of the most fatal and least understood human malignancies and continues to be a major unsolved health problem (Melisi et al, 2014). It has a five-year relative survival rate at $7.7 \%$ (SEER Stat Fact Sheets), and is projected to become the second leading cause of cancer-related death by the year 2030 in western countries (Rahib et al, 2014). More than 338000 people per year are diagnosed with pancreatic cancer worldwide, and approximately the same number die of this disease (Ferlay et al, 2013). The poor prognosis associated with pancreatic cancer can be attributed to the disease's early metastatic behaviour during progression, its aggressive course, and, in particular, to the limited efficacy of currently approved classic chemotherapeutic treatments (Tamburrino et al, 2013). Current treatment guidelines - most notably those from the National Comprehensive Cancer Network (version 2, 2016) and the European Society for Medical Oncology (ESMO, 2015) recommend the following first-line treatment options for advanced pancreatic cancer depending on patients' performance status (measured by Eastern Cooperative Oncology Group (ECOG) scale): gemcitabine and nab-paclitaxel combination therapy or fluorouracil-leucovorin-irinotecan-oxaliplatin (FOLFIRINOX) regimen (ECOG 0 or 1 ), and these two regimens are preferred chemotherapy as recommended in National Comprehensive Cancer Network and other acceptable regimens include gemcitabine + erlotinib, gemcitabine + capecitabine, and so on; gemcitabine monotherapy (ECOG 2); or best supportive care (ECOG 3-4 or comorbidities). However, the majority of patients progress while on first-line therapy and have limited proven options in the second-line setting. National Comprehensive Cancer Network guideline(s) have suggested that fluoropyrimidine-based chemotherapy, such as 5-fluorouracil and leucovorin (5-FU/LV), is an acceptable second-line option among patients previously treated with gemcitabine-based therapy (Tempero et al, 2014; Ducreux et al, 2015; Oettle et al, 2015).

Nanoliposomal irinotecan (nal-IRI; US trade name Onivyde) was recently approved by the US Food and Drug Administration and the European Medicines Agency review for the treatment of metastatic pancreatic adenocarcinoma (mPAC) in combination with $5-\mathrm{FU} / \mathrm{LV}$ in patients previously treated with gemcitabinebased therapy. The US Food and Drug Administration approved nal-IRI + 5-FU/LV based on results from the NAPOLI-1 (NAnoliPOsomaLIrinotecan) clinical trial (NLM identifier: NCT01494506). NAPOLI-1 was a global, multicenter, open-label, Phase 3 trial that included adult metastatic pancreatic cancer patients whose disease had progressed following previous gemcitabine-based therapy (Wang-Gillam et al, 2016). The NAPOLI-1 trial demonstrated that the nal-IRI $+5-\mathrm{FU} / \mathrm{LV}$ significantly improved patients' median overall survival (OS) $(6.1$ vs 4.2 months, $\mathrm{HR}=0.67, P=0.012$ ) and median progression-free survival (3.1 vs 1.5 months, $\mathrm{HR}=0.56, P=0.0001$ ) compared with 5-FU/LV therapy alone. In its 2015 updated guidelines, ESMO indicated nal-IRI + 5-FU + folinic acid as the best second-line treatment option based on the currently available evidence for this patient population (Ducreux et al, 2015).

The present analysis aimed to compare the quality-adjusted time without symptoms of disease progression or toxicity (QTWiST) of nal-IRI + 5-FU/LV treatment over 5-FU/LV treatment alone using the NAPOLI-1 clinical trial data. The Q-TWiST method (Chen et al, 2015) integrates the quality and quantity of survival by partitioning survival time into three clinically relevant periods, which are assigned different quality-of-life weights (i.e., 'utilities'), including the periods in which patients (1) experienced toxicity (TOX) due to treatment, (2) did not experience any TOX and had not yet progressed (i.e., pre-progression time without adverse events (AEs)), and (3) experienced progression after disease recurrence. This approach facilitates comparison between treatments by penalising treatments with increased toxicities or shorter times to disease progression and rewarding those with lower TOX and longer progression-free and OS times (Cole and Gelber, 1995).

\section{MATERIALS AND METHODS}

Study cohorts. The study cohorts for this analysis were from the NAPOLI-1 clinical trial (Chen et al, 2015; Wang-Gillam et al, 2016). Patients were randomised into three treatment arms: nalIRI, 5FU/LV, and nal-IRI + 5-FU/LV (1:1:1 ratio). The primary end point was OS; secondary end points included progression-free survival, time to treatment failure, and tumour reponse. The study's full inclusion and exclusion criteria and findings can be found in the NAPOLI-1 primary publication (Wang-Gillam et al, 2016).

The primary Q-TWiST analysis was performed using the intent to treat population, and was supplemented with analyses in the per-protocol population (i.e., patients who received $\geqslant 80 \%$ of the protocol-defined treatment during the first 6 weeks of treatment) (Chen et al, 2015) and analyses in nine pre-specified subgroups: race (Asian), baseline Karnofsky performance status scores $(<90$, 90-100), time since treatment ( $<1.3$ months, $>1.3$ months), cancer stage at diagnosis (stage IV, other), and tumour location (head, other). The Q-TWiST analysis was performed specifically on the combination treatment arm (nal-IRI + 5-FU/LV) vs 5-FU/ LV. The current study focused on the treatment arms amended after safety data on the combination became available, which is consistent with the primary analysis (Chen et al, 2015).

Statistical analysis. The Q-TWiST method combines treatment benefits and risks into a single measure by partitioning survival time into three health states and subsequently assigning quality-oflife weights to the survival time in each state (Gelber et al, 1993). These three health states include time with grade $\geqslant 3$ TOX (i.e., allcause AEs), disease progression (REL), and time without disease progression symptoms or grade $\geqslant 3$ toxicity (TWiST). The restricted mean duration of each health state was calculated as the area under its Kaplan-Meier curve through 12 months of follow-up (Kaplan and Meier, 1958; Fairclough, 2010).

TOX time was defined as the period during which a patient experienced AEs between randomisation and progression. It was calculated as the total number of days between randomisation and progression in which each patient experienced grade $\geqslant 3$ all-cause adverse events (AEs; defined using the National Cancer Institute Common Terminology Criteria for Adverse Events). Any day with multiple AEs was only counted once, and the total number of TOX days were grouped together during a patient's treatment, regardless of when TOX days occurred (but only counting AEs prior to relapse). REL time was defined as the time after disease progression (i.e., the period when patients experience disease relapse).

The Q-TWiST value is the linear combination of the health state durations adjusted by the respective utilities (U):

$$
\mathrm{Q}-\mathrm{TWiST}=\mathrm{U}(\mathrm{TOX}) \times \mathrm{TOX}+\mathrm{U}(\mathrm{TW} \text { iST }) \times \mathrm{TWiST}+\mathrm{U}(\mathrm{REL}) \times \mathrm{REL}
$$

Within this analysis, two assumptions about TOX time were made: (1) the utility associated with TOX was the same regardless of AE type or grade; (2) AE duration was truncated on the date the patient progressed if the $\mathrm{AE}$ continued past that date.

In the base case, the utility of TOX and REL were both assigned as 0.5 and TWiST was assigned as 1.0 consistent with prior Q-TWiST analyses in various oncology disease areas (Solem et al, 2014). In a threshold analysis, the difference in Q-TWiST between treatments was calculated over all possible combinations of $\mathrm{U}(\mathrm{TOX})$ and $\mathrm{U}(\mathrm{REL})$, ranging from 0.0 to 1.0. To understand how the utility weight of TWiST affects the results, alternative utility weights were used in a sensitivity analysis: 
$\mathrm{U}(\mathrm{TWiST})=0.80, \quad \mathrm{U}(\mathrm{TOX})=0.65$, and $\mathrm{U}(\mathrm{REL})=0.75$. These alternative utility weights of TWiST and REL were obtained from a clinical trial, which prospectively collected utility data of patients with mPAC (Romanus et al, 2012) who received gemcitabine monotherapy or gemcitabine-based combination therapy. The utility weight of TOX was calculated based on a series of study results reporting the utility decrement of AEs (Lloyd et al, 2006; Nafees et al, 2008; Swinburn et al, 2010; Tolley et al, 2013; Goldstein et al, 2014). Additionally, since afebrile neutropenia may not be clinically symptomatic, a sensitivity analysis was performed to assess the Q-TWiST difference when removing the $\mathrm{AE}$ of neutropenia from TOX time. Given that certain grade 2 toxicities also impact patients' quality of life, such as fatigue, a sensitivity analysis was performed by including grade 2 toxicities in the TOX time calculation.

Consistent with Revicki et al (2006), the relative gain in Q-TWiST in nal-IRI + 5-FU/LV over 5-FU/LV was calculated as the difference in Q-TWiST divided by the OS time of 5-FU/LV group (i.e., control group). It was reported for the base case Q-TWiST analysis and threshold analysis. The relative gain in Q-TWiST was used to define clinical important difference (10\%) and clearly clinically important difference (15\%) of Q-TWiST in Revicki et al.

Statistical testing was limited to a comparison of the overlap of 95\% confidence intervals (CIs), which were calculated using 1000 sample nonparametric bootstraps of the data (Fairclough, 2010). Mean durations of each health state and quality-adjusted survival time were compared and differences calculated between treatment groups. If the $95 \%$ CI did not overlap between treatment groups (or the 95\% CI around the difference did not overlap 0), the difference was considered statistically significant. SAS version 9.4 (SAS Institute, Inc., Cary, NC, USA) was used to perform the statistical analyses.

\section{RESULTS}

Patient sample. A total of 236 patients were randomised to nal$\mathrm{IRI}+5-\mathrm{FU} / \mathrm{LV}$ treatment $(n=117)$ or $5-\mathrm{FU} / \mathrm{LV}$ treatment $(n=119)$. Patients randomised to nal-IRI $+5-\mathrm{FU} / \mathrm{LV}$ had a mean (s.d.) age of 63.2 (9.06) years and were 59\% male and $29.1 \%$ Asian, while 5-FU/LV patients were aged 61.0 (9.46) years, 56.3\% male, and $30.3 \%$ Asian (Table 1). There were no significant differences in baseline characteristics between treatment groups.

Duration of time spent in each health state. The mean duration of time spent in TWiST, TOX, and REL was significantly longer among patients receiving nal-IRI + 5-FU/LV vs 5FU/LV alone (Table 2). In both cohorts, the mean TWiST duration was significantly higher than the mean TOX duration, indicating a greater amount of time without significant symptoms prior to progression compared to time with AEs.

Q-TWiST (NAL-IRI + 5-FU/LV vs 5-FU/LV). In the base case, when the utility weights for the TOX and REL health states were set to 0.5 in the intent to treat population, there was a statistically significant 1.3-month gain (95\% CI, 0.4-2.1 months) in Q-TWiST favouring nal-IRI + 5-FU/LV (5.1 months (95\% CI, 4.5-5.8 months)) over 5-FU/LV alone (3.9 months (95\% CI, 3.3-4.5 months)). This translated to a relative improvement of $23.8 \%$ at 12-month follow-up. In the per-protocol population, the results remained in favour of nal-IRI $+5-\mathrm{FU} / \mathrm{LV}$ over $5-\mathrm{FU} / \mathrm{LV}$ alone (difference in Q-TWiST: 1.8 months (95\% CI, 0.7-3.0 months)) (Table 3).

In the threshold analysis, the absolute gain in Q-TWiST at 12month follow-up showed that the gain in Q-TWiST increased from 0.9 to 1.7 months as $\mathrm{U}(\mathrm{TOX})$ and $\mathrm{U}(\mathrm{REL})$ increased from 0.0 to 1.0 (Figure 1A). The Q-TWiST gains were all statistically significant across all combinations of utilities in TOX and REL while TWiST was set at 1.0, except when utility of REL was close to 1.0 and utility of AE was close to 0.0 . Similarly, the relative improvement ranged from 16.9 to $30.8 \%$ (Figure $1 \mathrm{~B}$ ).

In a sensitivity analysis applying literature-based $\mathrm{mPAC}$ utilities to each health state $(\mathrm{U}(\mathrm{TOX})=0.65, \mathrm{U}(\mathrm{REL})=0.75$, and $\mathrm{U}(\mathrm{TWiST})=0.80)$, the Q-TWiST gain in nal-IRI + 5-FU/LV over $5-\mathrm{FU} / \mathrm{LV}$ remained statistically significant at 1.1 months $(95 \% \mathrm{CI}$, 0.3-1.9 months), with a relative gain of $20 \%$. Another sensitivity analysis was performed by removing neutropenia from the TOX time calculation, and the result remained similar to the main analysis (difference in Q-TWiST: 1.3 months (95\% CI, 0.4-2.2 months)). The Q-TWiST at various time points during follow-up consistently showed significant improvement in the nal-IRI +5 FU/LV group over the 5-FU/LV group, except at the first 3 months (Figure 2).

In another sensitivity analysis including grade $\geqslant 2$ toxicities, it was found that patients treated with nal-IRI $+5-\mathrm{FU} / \mathrm{LV}$ experienced longer quality-adjusted survival time compared to 5-FU/LV (difference in Q-TWiST: 0.9 month (95\% CI: 0.1, 1.7)), which corresponds to a $17.5 \%$ improvement. This sensitivity analysis result remained consistent with the main analysis, which included grade $\geqslant 3$ toxicities.

In subgroup analyses, the results across all pre-specified subgroups were in favour of nal-IRI +5 -FU/LV over 5 -FU/LV alone (Figure 3 ). These positive differences were significant for patients of Asian ethnicity, patients with Karnofsky performance status scores $<90,<1.3$ months since prior treatment, stage IV cancer at diagnosis, and tumour location other than the head of pancreas.

\section{DISCUSSION}

This analysis is the first to use the Q-TWiST approach to assess the efficacy of nal-IRI +5 -FU/LV combination treatment $v s 5$-FU/LV treatment alone in patients with metastatic pancreatic cancer. Within the NAPOLI-1 Q-TWiST analyses, nal-IRI in combination with 5-FU/LV provided significantly greater quality-adjusted survival time compared to 5-FU/LV alone in patients previously treated with gemcitabine-based therapy. This result reflects both the significantly greater median survival time (6.1 vs 4.2 months; $\mathrm{HR}=0.67(95 \%$ CI $0.49-0.92), P=0.012)$ and progression-free survival time (3.1 vs 1.5 months $\mathrm{HR}=0.56$ (95\% CI $0.41-0.75$ ), $P=0.0001)$ observed for nal-IRI +5 -FU/LV treatment over 5-FU/ LV alone within the NAPOLI-1 trial (Wang-Gillam et al, 2016). This also supports the original trial's finding that despite patients receiving more medicine in the combination arm, quality of life (measured using the European Organization for Research and Treatment of Cancer Quality-of-Life Core Questionnaire (EORTCQLQ-C30)) was not appreciably different between these two treatment groups (Wang-Gillam et al, 2016).

The Q-TWiST findings were consistent across the threshold analysis and various sensitivity and subgroup analyses. Most clinical trials do not collect utility data by health states, such as toxicities, as described in this study. The threshold analysis in Q-TWiST method allows TOX and post-progression utilities to vary across their possible ranges (0-1), addressing some of the uncertainty associated with the assigned utility values. Additionally, these Q-TWiST data may help inform physicians in discussing quality-of-life issues with pancreatic cancer when considering second-line chemotherapy in patients previously treated with gemcitabine-based therapy. In this study, the absolute gain and relative gain in Q-TWiST ranged from 0.9 to 1.7 months and from 17 to $31 \%$ in the threshold analysis, respectively. The results confirmed the robustness of the results given varying utilities 
Table 1. Study populations and demographics

\begin{tabular}{|c|c|c|}
\hline Characteristic & nal-IRI + 5-FU/LV combo $(n=117)$ & 5-FU/LV combo control $(n=119)$ \\
\hline $\begin{array}{l}\text { Age (y) } \\
\text { Mean (s.d.) } \\
\text { Median } \\
\text { Min, max }\end{array}$ & $\begin{array}{l}63.2(9.06) \\
63.0 \\
41,81\end{array}$ & $\begin{array}{c}61.0(9.46) \\
62.0 \\
34,80\end{array}$ \\
\hline $\begin{array}{l}\text { Gender, n (\%) } \\
\text { Female } \\
\text { Male }\end{array}$ & $\begin{array}{l}48(41.0) \\
69(59.0)\end{array}$ & $\begin{array}{l}52(43.7) \\
67(56.3)\end{array}$ \\
\hline $\begin{array}{l}\text { Baseline KPS level, n (\%) } \\
50 \\
60 \\
70 \\
80 \\
90 \\
100\end{array}$ & $\begin{aligned} 1 & (0.9) \\
2 & (1.7) \\
7 & (6.0) \\
38 & (32.5) \\
51 & (43.6) \\
18 & (15.4)\end{aligned}$ & $\begin{array}{c}0 \\
0 \\
10(8.4) \\
51(42.9) \\
40(33.6) \\
17(14.3)\end{array}$ \\
\hline $\begin{array}{l}\text { Baseline albumin, } \mathrm{g} \mathrm{dl}^{-1} \\
\text { Mean (s.d.) } \\
\text { Min, max }\end{array}$ & $\begin{array}{c}3.97(0.459) \\
2.6,5.1\end{array}$ & $\begin{array}{c}3.98(0.506) \\
2.4,5.0\end{array}$ \\
\hline Measurable lesions at baseline, $\mathrm{n}(\%)$ & $113(96.6)$ & $114(95.8)$ \\
\hline No measurable lesions at baseline, $\mathrm{n}(\%)$ & $4(3.4)$ & $5(4.2)$ \\
\hline Measurable metastatic lesions at baseline, $\mathrm{n}(\%)$ & $97(82.9)$ & $103(86.6)$ \\
\hline No measurable metastatic lesions at baseline, n (\%) & $20(17.1)$ & $16(13.4)$ \\
\hline $\begin{array}{l}\text { Subjects at baseline with: } \mathrm{n}(\%) \\
\text { One measurable metastatic lesion } \\
\text { Two measurable metastatic lesions } \\
\text { Three measurable metastatic lesions } \\
\text { > Three measurable metastatic lesions }\end{array}$ & $\begin{aligned} 19 & (16.2) \\
49 & (41.9) \\
22 & (18.8) \\
7 & (6.0)\end{aligned}$ & $\begin{array}{l}22(18.5) \\
58(48.7) \\
15(12.6) \\
8(6.7)\end{array}$ \\
\hline $\begin{array}{l}\text { Prior lines of treatment } \\
\text { First-line advanced/metastatic } \\
\text { Second-line advanced/metastatic } \\
\text { Third-line advanced/metastatic }\end{array}$ & $\begin{array}{l}15(12.8) \\
62(53.0) \\
40(34.2)\end{array}$ & $\begin{array}{l}15(12.8) \\
67(56.3) \\
37(31.1)\end{array}$ \\
\hline $\begin{array}{l}\text { Time since last prior anticancer therapy (months) } \\
\text { Mean (s.d.) } \\
\text { Median } \\
\text { Min, max }\end{array}$ & $\begin{array}{l}2.1(2.4) \\
1.4 \\
0.2,16.8\end{array}$ & $\begin{array}{c}2.6(5.4) \\
1.1 \\
0.0,43.2\end{array}$ \\
\hline $\begin{array}{l}\text { Location of pancreatic tumour of diagnosis, n (\%) } \\
\text { Head } \\
\text { Other }\end{array}$ & $\begin{array}{l}76(64.9) \\
41(35.1)\end{array}$ & $\begin{array}{l}69(58.0) \\
50(42.0)\end{array}$ \\
\hline $\begin{array}{l}\text { Disease stage, n (\%) } \\
\text { Stage I } \\
\text { Stage II } \\
\text { Stage III } \\
\text { Stage IV } \\
\text { Missing }\end{array}$ & $\begin{aligned} & 2(1.8) \\
32 & (27.3) \\
21 & (17.9) \\
61 & (52.1) \\
1 & (0.9)\end{aligned}$ & $\begin{array}{c}5(4.2) \\
31(26.1) \\
19(16.0) \\
62(52.1) \\
2(1.7)\end{array}$ \\
\hline
\end{tabular}

assigned to TOX and disease symptoms or progression. Additional sensitivity analysis was performed using alternative literature utilities (i.e., prospectively collected utilities in pancreatic cancer patients): Utility for TWiST was 0.8 , REL was 0.75 , and TOX was 0.65 . The results showed a statistically significant and $20 \%$ relative Q-TWiST gain for nal-IRI + 5-FU/LV vs 5-FU/LV, which further supports the study conclusion. Five out of the nine subgroups had significant Q-TWiST gains by nal-IRI + 5-FU/LV vs 5-FU/LV, including baseline Karnofsky performance status $<90$, time since treatment at baseline $<1.3$ months, stage IV cancer, Asian ethnicity, and tumour location other than head. It showed that patients with poor performance status or more advanced disease at 
Table 2. Mean duration of TOX, TWiST, and REL between treatment groups at 12 months for nal-IRI + 5-FU/LV vs 5-FU/ LV in the ITT cohort

\begin{tabular}{|c|c|c|c|}
\hline $\begin{array}{l}\text { Survival duration } \\
\text { (mo) by health } \\
\text { states }\end{array}$ & $\begin{array}{l}5-\mathrm{FU} / \mathrm{LV} \text {, } \\
\text { mean } \\
(95 \% \mathrm{Cl})\end{array}$ & $\begin{array}{l}\text { nal-IRI + 5-FU/ } \\
\text { LV, mean } \\
(95 \% \mathrm{CI})\end{array}$ & $\begin{array}{c}\text { Difference, } \\
\text { mean }(95 \% \mathrm{Cl})\end{array}$ \\
\hline TOX & $0.3(0.2,0.5)$ & $1.0(0.7,1.3)$ & $0.5(0.3,1.0)^{a}$ \\
\hline TWiST & $2.4(1.8,2.9)$ & $3.4(2.6,4.1)$ & $1.1(0.1,1.9)^{a}$ \\
\hline REL & $2.7(2.1,3.3)$ & $2.5(2.0,3.1)$ & $-0.1(-0.9,0.7)$ \\
\hline \multicolumn{4}{|c|}{ 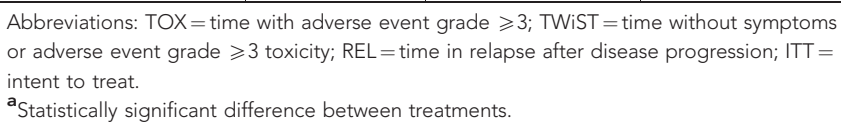 } \\
\hline
\end{tabular}

Table 3. Quality-adjusted time without symptoms or toxicities threshold analysis for nal-IRI + 5-FU/LV vs 5-FU/LV in the ITT cohort

\begin{tabular}{|c|c|c|c|}
\hline Utility weights & $\begin{array}{c}5-\mathrm{FU} / \mathrm{LV} \text {, } \\
\text { mean } \\
\text { Q-TWiST } \\
\text { months } \\
(95 \% \mathrm{Cl})\end{array}$ & $\begin{array}{c}\text { nal-IRI + 5- } \\
\text { FU/LV, } \\
\text { mean } \\
\text { Q-TWiST } \\
\text { months } \\
(95 \% \mathrm{Cl})\end{array}$ & $\begin{array}{l}\text { Difference, } \\
\text { mean Q-TWiST } \\
\text { months }(95 \% \mathrm{Cl})\end{array}$ \\
\hline $\mathrm{U}(\mathrm{TOX})=0, \mathrm{U}(\mathrm{REL})=0$ & $2.4(1.8,2.9)$ & $3.4(2.6,4.1)$ & $1.0(0.1,1.9)$ \\
\hline $\mathrm{U}(\mathrm{TOX})=0, \mathrm{U}(\mathrm{REL})=0.5$ & $3.7(3.1,4.3)$ & $4.7(4,5.3)$ & $1.0(0.1,1.8)$ \\
\hline $\mathrm{U}(\mathrm{TOX})=0, \mathrm{U}(\mathrm{REL})=1$ & $5(4.3,5.8)$ & $5.9(5.2,6.6)$ & $0.9(-0.2,2.0)$ \\
\hline$U(T O X)=0.5, U(R E L)=0$ & $2.5(2,3.1)$ & $3.9(3.1,4.5)$ & $1.3(0.4,2.2)$ \\
\hline$U(T O X)=0.5, U(R E L)=0.5$ & $3.9(3.3,4.5)$ & $5.1(4.5,5.8)$ & $1.3(0.4,2.1)$ \\
\hline $\mathrm{U}(\mathrm{TOX})=0.5, \mathrm{U}(\mathrm{REL})=1$ & $5.2(4.4,6)$ & $6.4(5.7,7.1)$ & $1.2(0.2,2.2)$ \\
\hline $\mathrm{U}(\mathrm{TOX})=1, \mathrm{U}(\mathrm{REL})=0$ & $2.7(2.1,3.3)$ & $4.4(3.6,4.9)$ & $1.7(0.7,2.5)$ \\
\hline $\mathrm{U}(\mathrm{TOX})=1, \mathrm{U}(\mathrm{REL})=0.5$ & $4(3.4,4.6)$ & $5.6(4.9,6.2)$ & $1.6(0.7,2.5)$ \\
\hline $\mathrm{U}(\mathrm{TOX})=1, \mathrm{U}(\mathrm{REL})=1$ & $5.4(4.6,6.2)$ & $6.9(6.1,7.6)$ & $1.5(0.4,2.5)$ \\
\hline \multicolumn{4}{|c|}{$\begin{array}{l}\text { Abbreviations: } \mathrm{U}(\mathrm{TOX})=\text { utility of time with adverse event grade } \geqslant 3 ; \mathrm{U}(\mathrm{REL})=\mathrm{utility} \text { of time } \\
\text { in relapse after disease progression; } \mathrm{Q}-\mathrm{TW} \text { iST }=\text { quality-adjusted time without symptoms of } \\
\text { disease progression or toxicity; ITT = intent to treat. }\end{array}$} \\
\hline
\end{tabular}

baseline were more likely to benefit in Q-TWiST from the treatment of nal-IRI + 5-FU/LV ( $v s 5$-FU/LV).

The statistically significant Q-TWiST gains are relatively large in magnitude and may be considered clearly clinically important using the thresholds established by Revicki et al (2006), which state a clinically important improvement for Q-TWiST as $10 \%$ of OS in the control group, while $15 \%$ indicates a clearly clinically important improvement. In contrast, Solem et al (2014) found in a recent review of Q-TWiST literature in oncology that only $18 \%$ of Q-TWiST studies reported a clearly clinically important gain (i.e., $\geqslant 15 \%$ ) in treatment over control groups in base case analyses.

A recent analysis assessing nab-paclitaxel plus gemcitabine combination treatment $v s$ gemcitabine-only in first-line mPAC (Reni et al, 2014) found a $21 \%$ relative improvement in Q-TWiST in favour of the combination treatment over gemcitabine-only treatment. The Q-TWiST improvement observed in the current analysis for nal-IRI + 5-FU/LV ( $v$ s 5-FU/LV) was 24\%. The difference in outcomes may be partially attributable to the study design and treatments; while Reni et al (2014) analysed first-line treatment on treatment-naive patients, the current analyses only included patients previously treated with gemcitabine-based regimen.

The present analysis has several limitations. First, patientderived data regarding the utility weights were not collected prospectively. To account for this, the base case utilities for TOX
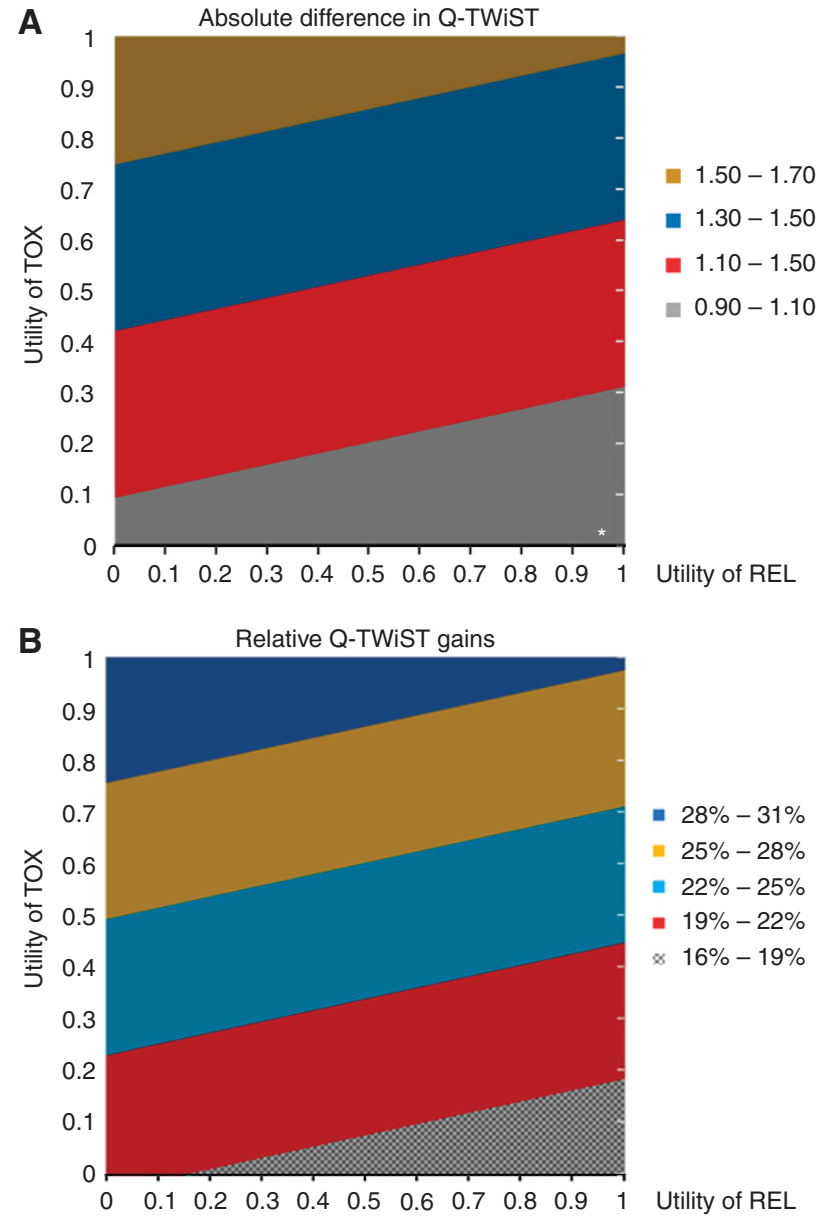

Figure 1. Utility threshold plot for Q-TWiST (nal-IRI + 5-FU/LV vs 5-FU/ LV) at 12 months in intent to treat cohort. (A) The $x$-axis represents the utility for time after disease progression (REL) and $y$-axis represents the utility for TOX time. Both $U(T O X)$ and $U(R E L)$ vary from 0 to 1 , with $\mathrm{U}(\mathrm{TW}$ iST) held at 1. The diagonal bands of different colours represent varying Q-TWiST gains by level of utility for TOX and REL. To understand the Q-TWiST gain associated with a given combination of $U(R E L)$ and $U(T O X)$, one must select the corresponding values of $U(R E L)$ and $U(T O X)$ on the $x$-axis and $y$-axis, respectively. The intersection of these two values inside the plot indicates which band of Q-TWiST gain the result from this combination belong. (B) The $x$-axis represents the utility for time after disease progression (REL) and $y$-axis represents the utility for TOX time. Both $U(T O X)$ and $U(R E L)$ vary from 0 to 1 , with U(TWiST) held at 1 . The diagonal bands of different colour represent varying relative $\mathrm{Q}-\mathrm{TW}$ SST gains by level of utility for TOX and REL, calculated by dividing the absolute Q-TWiST gain by the mean survival in the 5-FU/LV group. To understand relative Q-TWiST gain associated with a given combination of $U(R E L)$ and $U(T O X)$, one must select the corresponding values of $U(R E L)$ and $U(T O X)$ on the $x$-axis and $y$-axis, respectively. The intersection of these two values inside the plot indicates which band of relative Q-TWiST gain the result from this combination belong. *Not significant when utility of relapse is close to 1 and utility of $A E$ is close to 0 . REL = relapse; $T O X=$ toxicity.

and REL were both set to 0.5 , consistent with numerous previous studies. A utility threshold analysis was also applied with additional sensitivity analyses to assess how the Q-TWiST value would change under alternate assumption scenarios of individual patient preference. Second, the same utility was assumed regardless of the severity and type of $\mathrm{AE}$ (provided grade was $\geqslant 3$ ). Neutropenia is one of the most common AEs observed in the clinical trial and it is often asymptomatic in nature. Therefore, additional sensitivity 


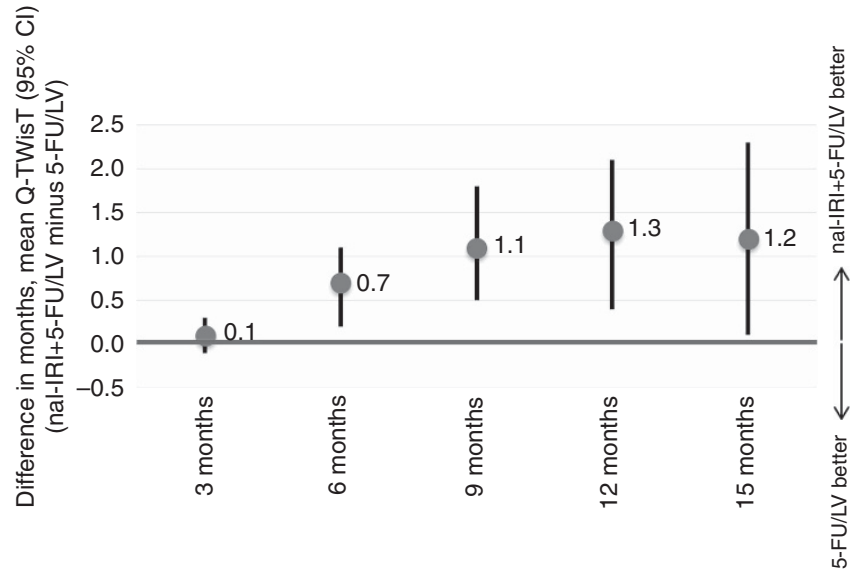

Figure 2. Differences in Q-TWiST between nal-IRI+5-FU/LV vs 5-FU/LV at 3, 6, 9, 12 and 15 Months of Follow-Up.

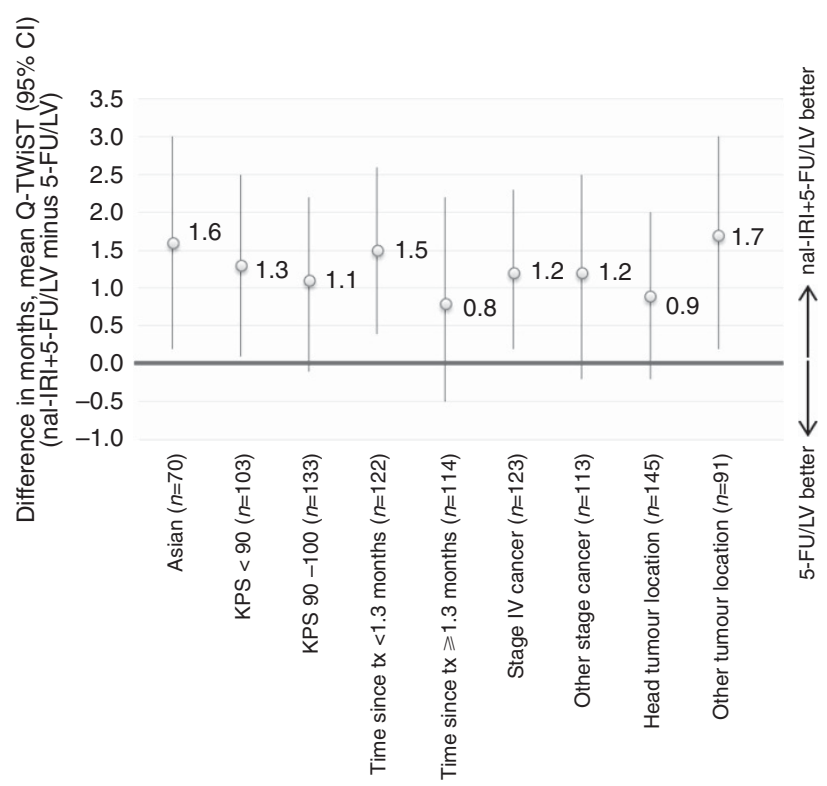

Figure 3. Quality-adjusted time without symptoms of disease progression or toxicity differences in months (5-FU/LV vs nal-IRI +5 -FU/LV) among subgroups. KPS $=$ Karnofsky performance status; $\mathrm{tx}=$ treatment.

analysis with and without neutropenia was calculated, which revealed negligible differences in results.

\section{CONCLUSION}

Applying the Q-TWiST methods to the NAPOLI-1 trial data, the combination of nal-IRI with 5-FU/LV resulted in a statistically significant and clinically meaningful gain in quality-adjusted survival vs 5-FU/LV alone. The results remained robust regardless of all possible combinations of utility weights assigned to the TOX time and disease symptom/progression time, which confirmed the favourable clinical risk benefit of nal-IRI + 5-FU/LV in mPAC patients previously treated with a gemcitabine-based therapy. In addition, the magnitude of Q-TWiST gains (i.e., relative gains) is relatively large when compared to published Q-TWiST analyses in other cancers, and is considered clearly clinically important based on the published thresholds.

\section{CONFLICT OF INTEREST}

UP has no disclosures. J-FB has received honoraria for lectures and consultancy and travel grants from Amgen, Bayer, Celgene, Merck, Roche and Sanofi-Aventis. J-FB has had a consultant or advisory role at Merck, Amgen, and Lilly. DM is a consultant for Lilly, and has received travel, accommodations, or expenses from Lilly and Celgene. AC has no disclosures. DDVH is employed by McKesson, and has had a consultant or advisory role at Arsia Therapeutics, Bionomics, Bristol-Myers Squibb, Cavion, Cerulean Pharma, CytRX Corporation, Dalian Wanchun Biotechnology, DNAtrix, Esperance Pharmaceuticals, Five Prime Therapeutics, Formula Pharmaceuticals, HealthCare Pharmaceuticals, Ignyta, Imaging Endpoints, Immodulon Therapeutics, Inform Genomics, Insys Therapeutics, Lilly, Medelis, Medical Prognosis Institute, Merus, miRNA Therapeutics, NuCana BioMed, Oncolytics Biotech, Pain Therapeutics, Pharmacyclics, Samumed, Senhwa Biosciences, Superlab Far East, Theravance, Tolero Pharmaceuticals, Toray Industries, Trovagene, Alethia Biotherapeutics, Alpha Cancer Technologies, Arvinas. Bellicum Pharmaceuticals, Biological Dynamics, CanBas, Horizon Discovery, Innate Pharma, Kinex, Lixte Biotechnology, MBC Pharma, Oncolyze, RenovoRx, Samsung, TD2, AADi, Aptose Biosciences, BiolineRx, CV6 Therapeutics, CytomX Therapeutics, Deciphera, EMD Serono, Evelo Therapeutics, Fujifilm, Gilead Sciences, Histogen, Horizon Pharma, Idera, Intezyne Technologies, Kalos Therapeutics, Kura, Lantern Pharma, Pain Therapeutics, Pfizer, Pharmamab, Phosplatin Therapeutics, Progen, SOTIO, Strategia Therapeutics, Sun Biopharma, Systems Imagination, Ventana Medical Systems. DDVH has received travel, accommodations, or expenses from FORMA Therapeutics and Merrimack. DDVH's patents, royalties, and other intellectual property include: intramedullary catheter, methods of human prostate cancer, use of 5,6-dihydro-5azacytidine in the treatment of prostate cancer, targeting site- 2 protease ( $\mathrm{S} 2 \mathrm{P}$ ) for the treatment of pancreatic cancer (pending), targeting ecto-5-nucleotidase (CD73) for the treatment of pancreatic cancer, targeting a protein tyrosine phosphotase-PRL-1 for the treatment of pancreatic cancer (pending), targeting a protein $\mathrm{PRC1}$ for the treatment of pancreatic cancer (pending), protein kinase inhibitors (pending), methods, compounds and compositions with genotype selective anticancer activity (pending), methods and kits to predict therapeutic outcome of BTK inhibitors (pending), muscle fatigue substance cytokines and methods of inhibiting tumour growth therewith (pending), 2-arylpyridylazoles for the treatment of solid tumours such as pancreatic cancer (pending). DDVH has stock or ownership interests in cell therapeutics, McKesson, Medtronic, CerRx, EmergingMed, and SynDevRx. DDVH has received honoraria from Celgene, Cerulean Pharma, and Genentech. DDVH's institution has received research funding from Lilly, Genentech, Celgene, Agios, Incyte, Merrimack, Plexxikon, Minneamrita Therapeutics, 3-V Biosciences, Abbvie, Aduro Biotech, ArQule, Baxalta, Biomarin, Cleave Biosciences, CytRx Corporation, Daiichi Sankyo, Deciphera, Endocyte, Exelixis, ESSA, Five Prime Therapeutics, Gilead Sciences, Merck, miRNA Therapeutics, Pfizer, Pharmacyclics, Phoenix Biotech, Proderm IQ, Sammumed, Strategia, Trovagene, and Verastem. AW-G has had a consultant or advisory role at Merrimack and Pfizer. AW-G has received travel, accommodations, or expenses from Axis Pharma. AW-G received honoraria from Pfizer and Merrimack. AW-G's institution has received research funding from Newlink Genetics, Precision Therapeutics, AstraZeneca, Merrimack, Aduro Biotech, Prometheus, EMD Serono, Pfizer, Halozyme, OncoMed, and CTI. 
L-TC received honoraria from and has had a consulting or advisory role at PharmaEngine and Merrimack. JTS has had a consulting or advisory role at Merrimack, Lilly, Amcure, Baxalta (now Shire), and Celgene. JTS has received research funding from Novartis, Boehringer Ingelheim, and Celgene. YW is employed by Pharmerit. YW's institution has received consulting or advisory role and research funding from Baxalta (now Shire) and numerous other sponsors from the pharmaceutical industry. CTS is employed by Pharmerit. CTS's institution has received consulting or advisory role and research funding from Baxalta (now Shire) and numerous other sponsors from the pharmaceutical industry. MFB is employed by Pharmerit, and has stock or ownership interests in Pharmerit. MFB's institution has received consulting or advisory role and research funding from Baxalta (now Shire) and numerous other sponsors from the pharmaceutical industry. YY is employed by Baxalta (now Shire) and has stock or ownership interests in Baxalta (now Shire). FAdJ is employed by Baxalta (now Shire) and has stock or ownership interests in Baxalta (now Shire). RAH has had a consulting or advisory role with BTG and Celgene.

\section{REFERENCES}

Chen L-T, Von Hoff DD, Li C-P, Wang-Gillam A, Bodoky G, Dean AP, Shan Y-S, Jameson GS, Macarulla T, Lee K-H (2015) Expanded analyses of napoli-1: phase 3 study of MM-398 (nal-IRI), with or without 5 -fluorouracil and leucovorin, versus 5-fluorouracil and leucovorin, in metastatic pancreatic cancer (mPAC) previously treated with gemcitabinebased therapy. J Clin Oncol 33: 234.

Cole BF, Gelber RD (1995) A quality-adjusted survival meta-analysis of adjuvant chemotherapy for premenopausal breast cancer. Stat Med 14: 1771-1784.

Ducreux M, Cuhna AS, Caramella C, Hollebecque A, Burtin P, Goéré D, Seufferlein T, Haustermans K, Van Laethem J, Conroy T (2015) Cancer of the pancreas: ESMO clinical practice guidelines for diagnosis, treatment and follow-up. Ann Oncol 26: v56-v68.

Fairclough DL (2010) Design and Analysis of Quality of Life Studies in Clinical Trials. CRC Press.

Ferlay J, Steliarova-Foucher E, Lortet-Tieulent J, Rosso S, Coebergh JW, Comber H, Forman D, Bray F (2013) Cancer incidence and mortality patterns in Europe: estimates for 40 countries in 2012. Eur J Cancer 49(6): 1374-1403.

Gelber RD, Goldhirsch A, Cole BF. Group IBCS (1993) Evaluation of effectiveness: Q-TWiST. Cancer Treat Rev 19: 73-84.

Goldstein D, Maraghi E, Hassan R, Hammel P, Heinemann V, Kunzmann V, Sastre J, Scheithauer W, Siena S, Tabernero J (2014) Updated survival from a randomized phase III trial (MPACT) of nab-paclitaxel plus gemcitabine versus gemcitabine alone for patients (pts) with metastatic adenocarcinoma of the pancreas. ASCO Annu Meeting Proc 2014: 178.

Kaplan EL, Meier P (1958) Nonparametric estimation from incomplete observations. J Am Stat Assoc 53: 457-481.

Lloyd A, Nafees B, Narewska J, Dewilde S, Watkins J (2006) Health state utilities for metastatic breast cancer. BJC 95: 683-690.
Melisi D, Calvetti L, Frizziero M, Tortora G (2014) Pancreatic cancer: systemic combination therapies for a heterogeneous disease. Curr Pharm Des 20: 6660-6669.

Nafees B, Stafford M, Gavriel S, Bhalla S, Watkins J (2008) Health state utilities for non small cell lung cancer. Health Qual Life Outcomes 6: 1.

Oettle H, Riess H, Stieler JM, Heil G, Schwaner I, Seraphin J, Görner M, Mölle M, Greten TF, Lakner V, Bischoff S, Sinn M, Dörken B, Pelzer U (2015) Second-line oxaliplatin, folinic acid, and fluorouracil versus folinic acid and fluorouracil alone for gemcitabine-refractory pancreatic cancer: outcomes from the CONKO-003 trial. J Clin Oncol 32: 2423-2429.

Rahib L, Smith BD, Aizenberg R, Rosenzweig AB, Fleshman JM, Matrisian LM (2014) Projecting cancer incidence and deaths to 2030: the unexpected burden of thyroid, liver, and pancreas cancers in the United States. Cancer Res 74: 2913-2921.

Reni M, Wan Y, Solem C, Whiting S, Ji X, Botteman M (2014) Qualityadjusted survival with combination nab-paclitaxel + gemcitabine vs gemcitabine alone in metastatic pancreatic cancer: a Q-TWiST analysis. J Med Econ 17: 338-346.

Revicki DA, Feeny D, Hunt TL, Cole BF (2006) Analyzing oncology clinical trial data using the Q-TWiST method: clinical importance and sources for health state preference data. Qual Life Res 15: 411-423.

Romanus D, Kindler HL, Archer L, Basch E, Niedzwiecki D, Weeks J, Schrag D, Cancer BLG (2012) Does health-related quality of life improve for advanced pancreatic cancer patients who respond to gemcitabine? Analysis of a randomized phase III trial of the cancer and leukemia group B (CALGB 80303). J Pain Symptom Manage 43 205-217.

Solem C, Wan Y, Botteman M (2014) Systematic review of the Q-TWiST in oncology. Value Health 17: A194-A195.

Swinburn P, Lloyd A, Nathan P, Choueiri TK, Cella D, Neary MP (2010) Elicitation of health state utilities in metastatic renal cell carcinoma. Curr Med Res Opin 26: 1091-1096.

Tamburrino A, Piro G, Carbone C, Tortora G, Melisi D (2013) Mechanisms of resistance to chemotherapeutic and anti-angiogenic drugs as novel targets for pancreatic cancer therapy. Front Pharmacol 4: 56.

Tempero MA, Malafa MP, Behrman SW, Benson AB, Casper ES, Chiorean EG, Chung V, Cohen SJ, Czito B, Engebretson A (2014) Pancreatic adenocarcinoma, version 2.2014. J Natl Compr Canc Netw 12: 1083-1093.

Tolley K, Goad C, Yi Y, Maroudas P, Haiderali A, Thompson G (2013) Utility elicitation study in the UK general public for late-stage chronic lymphocytic leukaemia. Eur J Health Econ 14: 749-759.

Wang-Gillam A, Li C-P, Bodoky G, Dean A, Shan Y-S, Jameson G, Macarulla T, Lee K-H, Cunningham D, Blanc JF (2016) Nanoliposomal irinotecan with fluorouracil and folinic acid in metastatic pancreatic cancer after previous gemcitabine-based therapy (NAPOLI-1): a global, randomised, open-label, phase 3 trial. Lancet 387: 545-557.

This work is published under the standard license to publish agreement. After 12 months the work will become freely available and the license terms will switch to a Creative Commons Attribution-NonCommercial-Share Alike 4.0 Unported License. 